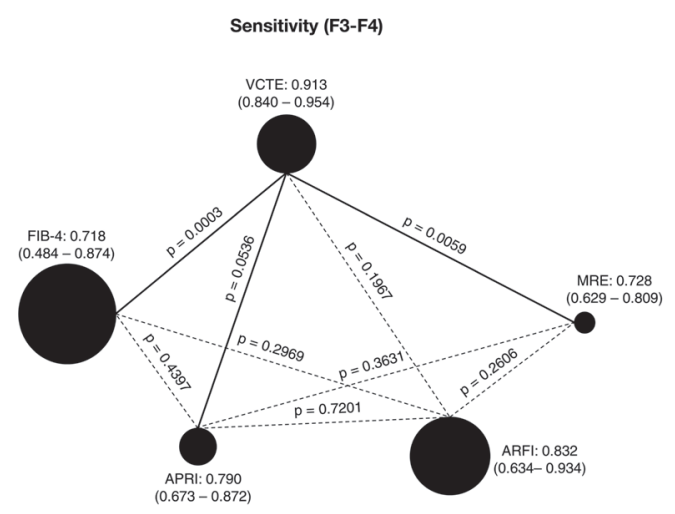

Abstract IDDF2021-ABS-0092 Figure 2

0092 Table 1). In brief, AFRI and VCTE provided sufficient diagnostic accuracy at F2 and F3. VCTE was additionally suited for F4 diagnosis. Blood-based NIT was found to be an inaccurate measure of fibrosis. When comparisons were conducted between NITs, there was no significant difference between VCTE and AFRI at F2 and F3 in both sensitivity and specificity (IDDF2021-ABS-0092 Figure 1, IDDF2021ABS-0092 Figure 2). Blood-based NIT was inferior to VCTE and AFRI.

Conclusions To our knowledge, our study is the first systematic evaluation of NITs after transplant. The diagnostic accuracy suggests that VCTE and ARFI should be employed as measures of fibrosis after LT. The presence of thrombocytopenia after LT results in the reduced accuracy of FIB-4 and ARFI. More studies are required to assess other measures of NITs, including MRE.

\section{IDDF2021-ABS-0095 A META-ANALYSIS AND SYSTEMATIC REVIEW ON THE GLOBAL PREVALENCE, RISK FACTORS AND OUTCOMES OF CORONARY ARTERY DISEASE IN LIVER TRANSPLANT RECIPIENTS}

${ }^{1}$ Cheng Han Ng*, 'Jieling Xiao, 'Jie Ning Yong, 'Nicholas Syn, 'Wen Hui Lim, 'Darren Jun Hao Tan, ${ }^{1}$ En Ying Tan, ${ }^{1}$ Daniel Huang, ${ }^{2}$ Raymond Wong, ${ }^{2}$ Nicholas Chew, ${ }^{1}$ Eunice Xiang Xuan Tan, ${ }^{3}$ Mazen Noureddin, ${ }^{1}$ Mark Dinesh Muthiah, ${ }^{4}$ Mohammad Shadab Siddiqui. ${ }^{1}$ Yong Loo Lin School of Medicine, Singapore; ${ }^{2}$ Department of Cardiology, National University Heart Centre, National University Hospital, Singapore; ${ }^{3}$ Cedars-Sinai Fatty Liver Program, Division of Digestive and Liver Diseases, Department of Medicine, Comprehensive Transplant Center, Cedars-Sinai Medical Center, Los Angeles, USA; ${ }^{4}$ Division of Gastroenterology, Hepatology and Nutrition, Department of Internal Medicine, Virginia Commonwealth University, Richmond, Virginia, USA

\subsection{6/gutjnl-2021-IDDF.88}

Background The changing demographics of waitlisted liver transplantation (LT) patients and shift in etiology of liver disease result in increasing prevalence of coronary artery disease (CAD), which can impact post-LT outcomes. This study aims to evaluate the prevalence of CAD among potential LT recipients, describe the risk factors of $\mathrm{CAD}$ and investigate the impact of pre-LT CAD on post-LT outcomes.

Methods Two electronic databases, MEDLINE and EMBASE, were searched for articles describing the CAD in pre-LT patients. Articles that describe the prevalence, risk factors and outcomes of pre-LT patients diagnosed with CAD were

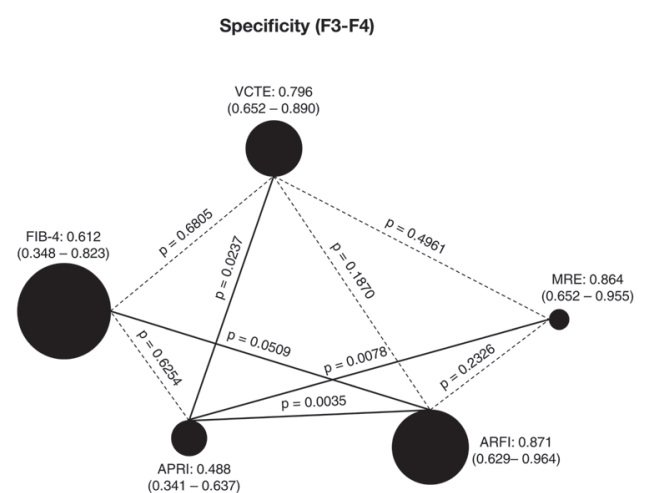

included. Generalized linear mixed model (GLMM) and Clopper-Pearson intervals to stabilize the variance were used to tabulate the prevalence of CAD in pre-LT patients and outcomes post-LT. The prevalence of CAD in pre-LT patients was stratified across different regions and countries of varying income levels. Risk factors of CAD and outcomes were analyzed with regression or pairwise analysis.

Results Total of 39 articles were included. The pooled prevalence of 15,880 patients from 34 studies diagnosed with CAD before LT was 15.88\% (CI: 9.81\% - 24.69\%, IDDF2021-ABS-0095 Figure 1. Screening Method of CAD before Liver Transplant). LT candidates in high-income countries had markedly higher odds of CAD (OR: 10.5; CI: 8.12 - 13.7; p < 0.0001) compared to patients in middle-income countries. Age, male, presence of diabetes, hypertension, hyperlipidemia, smoking, non-alcoholic steatohepatitis, hepatitis B and hepatocellular carcinoma were significantly associated with CAD. CAD diagnosis prior to LT was a significant risk factor for overall mortality (OR: 1.42; CI: $1.40-1.43$; $\mathrm{p}=0.0140$ ) and cardiac-related mortality (OR: 1.18; CI: $1.10-1.27 ; \mathrm{p}=0.0347)$ post-LT. North America had the highest pooled prevalence of CAD in pre-LT patients at 23.61\% (CI: 14.07\% - 36.83\%) amongst South America, Europe, Middle east, Asia and Oceania. 69\% of the included studies utilized non-invasive stress tests and invasive coronary angiograms in their pre-LT cardiac assessment (IDDF2021ABS-0095 Figure 2. Global Prevalence of CAD before Liver Transplant).

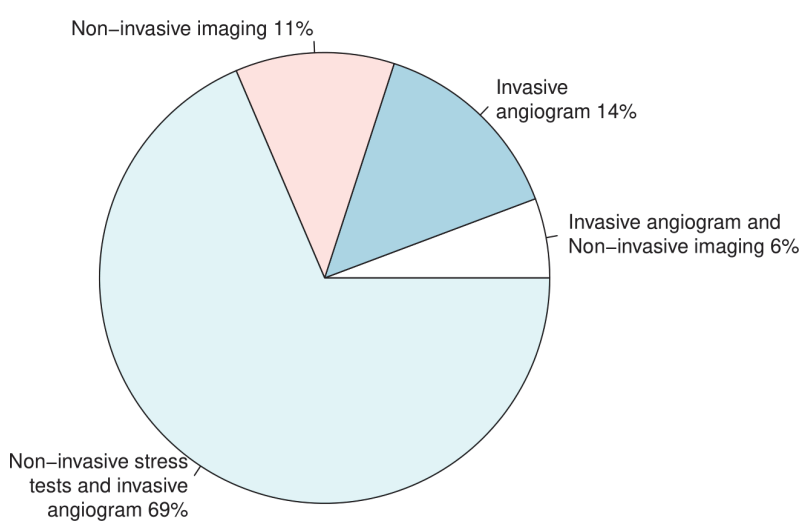

Abstract IDDF2021-ABS-0095 Figure 1 


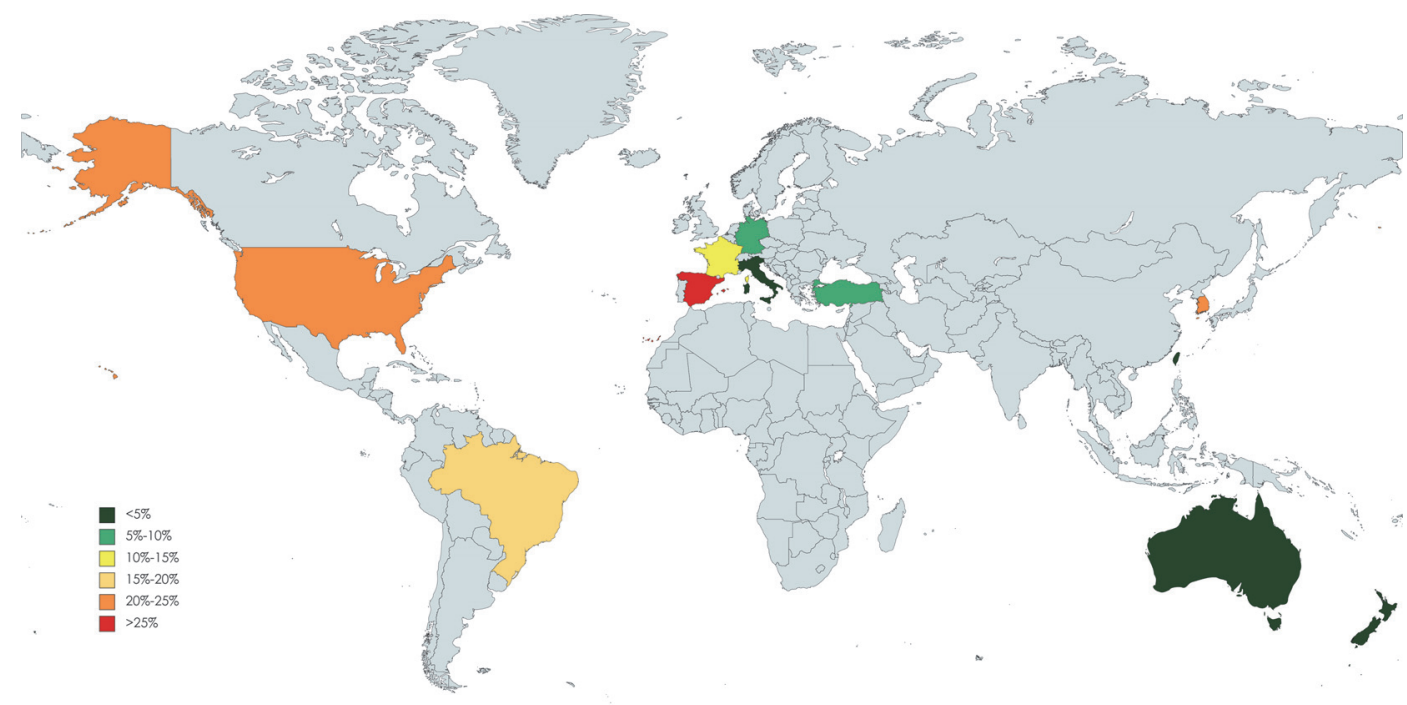

\section{Abstract IDDF2021-ABS-0095 Figure 2}

Conclusions This meta-analysis and systematic review demonstrate the high prevalence of CAD in pre-LT patients, the associated risk factors and outcomes. More studies are required to determine the optimal screening methodology for CAD in pre-LT patients.

\section{IDDF2021-ABS-0096 SELECTING ANTI-DIABETIC MEDICATIONS FOR TYPE 2 DIABETICS WITH NON- ALCOHOLIC FATTY LIVER DISEASE. EVIDENCE FROM A NETWORK META- ANALYSIS}

${ }^{1}$ Cheng Han Ng*${ }^{*}$, Snow Yunni Lin, ${ }^{1}$ Yip Han Chin, ${ }^{1}$ Ming Hui Lee, ${ }^{1}$ Nicholas Syn, ${ }^{1}$ Xin Lei Goh, 'Shao Feng Mok, 'Eunice Tan, 'Daniel Huang, ${ }^{2}$ Nicholas Chew, ${ }^{1}$ Chin Ming Khoo, ${ }^{1}$ Mark Dinesh Muthiah, ${ }^{3}$ Shadab Siddiqui. 'Y Yong Loo Lin School of Medicine, Singapore; ${ }^{2}$ Department of Cardiology, National University Hospital, Singapore; ${ }^{3}$ Department of Internal Medicine, Division of Gastroenterology, Hepatology and Nutrition, Virginia Commonwealth University, USA

\subsection{6/gutjnl-2021-IDDF.89}

Background Non-alcoholic fatty liver disease (NAFLD) is an increasingly common disease, where more than half of diabetic patients may develop NAFLD. Judicious selection of anti-diabetic agents is imperative to reduce mortality. Recent trials suggest that anti-diabetic drugs have great promise for the treatment of NAFLD. However, the comparative efficacy between individual agents remains unclear.

Methods We systematically searched Medline and Embase for studies on biguanides, sulfonylurea, peroxisome proliferatoractivated receptor gamma (PPAR $\gamma)$ agonists, insulin, sodiumglucose transport protein 2 inhibitors (SGLT2i) and glucagonlike peptide-1 receptor agonists (GLP1-RA) for the treatment of NAFLD in diabetic patients. We conducted a frequential network meta-analysis with $\mathrm{p}$-score as a surrogate measure of treatment effectiveness.

Results 13 studies were included and the p-score results can be found in IDDF2021-ABS-0096 Table 1. PPAR $\gamma$ agonists were most efficacious for reducing hepatic steatosis. PPAR $\gamma$ agonists (MD: $-6.02 \%, \mathrm{CI}:-10.37$ to -1.67 ), SGLT2i (MD: 2.60\%, CI: $-4.87 \%$ to $-0.33 \%$ ) and GLP1-RA (MD: $-3.50 \%$, CI: $-7.39 \%$ to $0.39 \%$ ) were superior to standard of care for reducing hepatic steatosis, although there was no significant difference when comparing GLP-1RA (MD: -0.90, CI: -5.41 to 3.61) and PPAR $\gamma$ agonists (MD: -3.42 , CI: -7.13 to 0.29 ) versus SGLT2i. SGLT2i was most effective for fibrosis reduction versus PPAR $\gamma$ agonists (MD: $-0.06, \mathrm{CI}$ : -0.10 to -0.02 ) and standard of care (MD: -0.13 , CI: -0.34 to 0.20$)$. GLP1RA yielded the greatest improvement in ALT and had greater efficacy versus standard of care (MD: -9.41 , CI: -19.46 to

Abstract IDDF2021-ABS-0096 Table 1 P-score summary of liver parameters with hypoglycaemic agents in NAFLD diabetes

\begin{tabular}{|c|c|c|c|c|c|c|c|c|c|c|}
\hline & MRI-PDFF & FIB-4 & AST & ALT & GGT & BMI & TC & TG & LDL & HDL \\
\hline Biguanide & - & - & 0.8305 & 0.761 & 0.9976 & 0.6892 & 0.4 & 0.0593 & 0.5486 & 0.307 \\
\hline GLP1-RA & 0.7 & - & 0.821 & 0.8434 & 0.8251 & 0.8395 & 0.232 & 0.4632 & 0.2231 & 0.5032 \\
\hline PPAR $\gamma$ agonists & 0.9372 & 0.3487 & 0.4669 & 0.6321 & 0.5375 & 0.0039 & 0.4871 & 0.5921 & 0.5121 & 0.7414 \\
\hline SGLT2I & 0.5784 & 0.914 & 0.4513 & 0.6987 & 0.3908 & 0.8764 & 0.7739 & 0.6204 & 0.8281 & 0.8394 \\
\hline Insulin Glargine & 0.063 & - & 0.386 & 0.2119 & 0.5282 & 0.3097 & 0.0199 & 0.5686 & 0.0094 & 0.5645 \\
\hline Standard of Care & 0.2214 & 0.2372 & 0.2352 & 0.2042 & 0.2052 & 0.5192 & 0.8769 & 0.6384 & 0.7828 & 0.4695 \\
\hline Sulfonylurea & - & - & 0.309 & 0.1487 & 0.0155 & 0.2621 & 0.7102 & 0.558 & 0.5959 & 0.075 \\
\hline
\end{tabular}

PPAR $\gamma$ - Peroxisome proliferator-activated receptor gamma, SGLT2I - Sodium-glucose co-transporter-2 inhibitors; GLP-RA - glucagon-like peptide-1 receptor agonists; AST - aspartate aminotransferase; ALT - alanine aminotransferase; GGT - Gamma-glutamyl transferase; MRI-PDFF - magnetic resonance imaging derived proton density fat fraction; Fibrosis-4 - FIB4; BMI - Body Mass Index; TC - Total Cholesterol; TG - Triglycerides; LDL - Low density lipoprotein; HDL - high density lipoprotein 